\title{
On sums of five almost equal prime squares
}

\author{
by \\ JiANYA LIU and TAO ZHAN (Jinan)
}

1. Introduction and statement of results. In 1938, Hua [6] proved that every sufficiently large integer $N \equiv 5(\bmod 24)$ is a sum of five prime squares. The purpose of this paper is to establish the following stronger result subject to the generalized Riemann hypothesis (GRH).

TheOREm 1. Assume GRH. Denote by $R(N, U)$ the number of solutions of the Diophantine equation with prime variables

$$
\left\{\begin{array}{l}
N=p_{1}^{2}+p_{2}^{2}+p_{3}^{2}+p_{4}^{2}+p_{5}^{2} \\
\left|p_{j}-\sqrt{N / 5}\right| \leq U, \quad j=1, \ldots, 5
\end{array}\right.
$$

Then for $U=N^{9 / 20+\varepsilon}$, we have

$$
R(N, U)=\frac{460 \sqrt{5}}{3} \sigma(N) \frac{U^{4}}{N^{1 / 2} \log ^{5} N}(1+o(1)),
$$

where

$$
\sigma(N)=\sum_{q=1}^{\infty} \frac{1}{\varphi^{5}(n)} \sum_{\substack{a=1 \\(a, q)=1}}^{q} C^{5}(a, q) e\left(-\frac{a N}{q}\right)
$$

with

$$
C(a, q)=\sum_{\substack{h=1 \\(h, q)=1}}^{q} e\left(\frac{a h^{2}}{q}\right) .
$$

Here $\sigma(N)$ is the so-called singular series, which is convergent and satisfies $\sigma(N)>c>0$ for $N \equiv 5(\bmod 24)$.

This result seems new of its kind. But there have already been great advances in related topics. For instance, Wright [14] in 1937 obtained cor-

1991 Mathematics Subject Classification: 11L07, 11P32.

Project supported by the Trans-Century Training Programme Foundation for Talents by the State Education Commission and the National Natural Science Foundation of China. 
responding results for Waring's problem. It is a well-known theorem of Lagrange that every positive integer is a sum of four squares. While Wright proved, among other things, that almost all large integers $N$ are expressible in the form

$$
\left\{\begin{array}{l}
N=n_{1}^{2}+n_{2}^{2}+n_{3}^{2}+n_{4}^{2}, \\
\left|n_{j}-\sqrt{N / 4}\right| \leq N^{3 / 10}, \quad j=1,2,3,4
\end{array}\right.
$$

where $n_{j}$ are positive integers, and an asymptotic formula holds for the number of representations. On the other hand, the problem of representing an odd integer as a sum of three almost equal primes has been attacked by a number of authors. For example, based on the work of the Pan brothers [10], Balog and Perelli [2], it was proved by Zhan [15] in 1991 that every large odd integer $N$ can be represented as

$$
\left\{\begin{array}{l}
N=p_{1}+p_{2}+p_{3} \\
\left|p_{j}-N / 3\right| \leq N^{5 / 8}(\log N)^{c}, \quad j=1,2,3,
\end{array}\right.
$$

and an asymptotic formula holds for the number of representations.

At the cost of the asymptotic formulae for the numbers of representations, the above results have been improved. For example, Auluck and Chowla [1] in 1937 showed that every large integer $N \not \equiv 0(\bmod 8)$ is expressible in the form of (1.1) with 3/10 replaced by 1/4. And Jia [7] in 1994 has reduced the exponent $5 / 8$ in $(1.2)$ to $7 / 12+\varepsilon$.

It seems that one should manage to prove Theorem 1 without GRH, just as in the related topics mentioned above. But deep investigation reveals that the problem dealt with in Theorem 1 is essentially different from and more difficult than the corresponding problems: the method used to treat the later problems can only give trivial result here. The new difficulty arising in this situation demands GRH as well as new estimates for exponential sums over primes, which will be given in Theorems 2 and 3 .

To see the new difficulty clearly, one follows the ideas in establishing (1.2), which suggest that the proof should start from the estimate of the exponential sum

$$
S_{2}(x, y ; \alpha)=\sum_{x<n \leq x+y} \Lambda(n) e\left(n^{2} \alpha\right)
$$

for all $\alpha \in[0,1]$. Such an estimate has been given in our previous papers ([8], [9]). Let $1 \leq y \leq x$ be real, $\Lambda(n)$ the von Mangoldt function, and $e(x)=e^{2 \pi i x}$ as usual. Suppose

$$
P_{0}=(\log x)^{c_{1}}, \quad R_{0}=y x(\log x)^{c_{2}},
$$

where $c_{1}, c_{2}$ are positive constants, and write $\alpha$ in the form

$$
\alpha=\frac{a}{q}+\lambda, \quad 1 \leq a \leq q,(a, q)=1 .
$$


Then the interval $[0,1]$ is the disjoint union of $F_{1}$ and $F_{2}$ defined as

$$
F_{1}=\left\{\alpha: q \leq P_{0},|\lambda| \leq 1 /\left(q R_{0}\right)\right\}, \quad F_{2}=[0,1]-F_{1} .
$$

It was proved in $[8]$ that the estimate

$$
S_{2}(x, y ; \alpha)= \begin{cases}M_{2}(x, y ; \alpha)+O\left(y(\log x)^{-A}\right), & \alpha \in F_{1}, \\ O\left(y(\log x)^{-A}\right), & \alpha \in F_{2},\end{cases}
$$

holds for $x^{11 / 16+\varepsilon} \leq y \leq x$, where $M_{2}(x, y ; \alpha)$ is the main term, which can be expressed as

$$
M_{2}(x, y ; \alpha)=\frac{1}{\varphi(q)} \sum_{\substack{h=1 \\(h, q)=1}}^{q} e\left(\frac{a h^{2}}{q}\right) \int_{x}^{x+y} e\left(\lambda u^{2}\right) d u .
$$

Thus the problem considered in Theorem 1 reduces to the estimation of

$$
I_{1}=\int_{F_{1}} S^{5}(\alpha) e(-N \alpha) d \alpha, \quad I_{2}=\int_{F_{2}} S^{5}(\alpha) e(-N \alpha) d \alpha,
$$

where

$$
S(\alpha)=\sum_{N_{1}<n \leq N_{2}} \Lambda(n) e\left(n^{2} \alpha\right)
$$

with

$$
N_{1}=\sqrt{\frac{N}{5}}-U, \quad N_{2}=\sqrt{\frac{N}{5}}+U .
$$

The first integral can be eventually calculated, on appealing to (1.6), as

$$
I_{1}=c_{3} \sigma(N) \frac{U^{4}}{N^{1 / 2}}(1+o(1))
$$

where $c_{3}>0$ is a suitable constant. While if we also apply (1.6) to estimate the second integral, we will arrive at

$$
I_{2} \ll\left\{\sup _{\alpha \in F_{2}}|S(\alpha)|\right\} \int_{F_{2}}|S(\alpha)|^{4} d \alpha \ll \frac{U}{(\log N)^{A}} \int_{0}^{1}|S(\alpha)|^{4} d \alpha .
$$

Now if we could show

$$
\int_{0}^{1}|S(\alpha)|^{4} d \alpha \ll U^{3} / N^{1 / 2},
$$

then Theorem 1 would hold for $U=N^{11 / 32+\varepsilon}$ without GRH. Unfortunately, such a result cannot be true, since one can easily verify that

$$
\int_{0}^{1}|S(\alpha)|^{4} d \alpha \gg U^{2} \gg U^{3} / N^{1 / 2},
$$

on noting that $U \leq N^{1 / 2}$. 
Therefore we need estimates of $S_{2}(x, y ; \alpha)$ different from (1.6). The secondary purpose of this paper is to give such estimates, which are formulated as Theorems 2 and 3.

Let $P, Q$ be parameters such that

$$
2 P^{2}<Q<x^{2}
$$

and $\alpha$ has the form of $(1.5)$. Then the interval $[0,1]$ is the disjoint union of $E_{1}$ and $E_{2}$ defined as

$$
E_{1}=\{\alpha: q \leq P,|\lambda| \leq 1 /(q Q)\}, \quad E_{2}=[0,1]-E_{1} .
$$

It therefore follows from Dirichlet's lemma on rational approximations that

$$
E_{2} \subset\{\alpha: P<q \leq Q,|\lambda| \leq 1 /(q Q)\} .
$$

The estimate of $S_{2}(x, y ; \alpha)$ on $E_{2}$ is contained in

Theorem 2. Let $\varepsilon>0$ be arbitrary. Then

$$
S_{2}(x, y ; \alpha) \ll y^{1+\varepsilon}\left(\frac{1}{q}+\frac{x^{1 / 2}}{y}+\frac{x^{4 / 3}}{y^{2}}+\frac{q x}{y^{3}}\right)^{1 / 4}
$$

holds for $\alpha$ satisfying

$$
1 \leq q \leq x y, \quad|\lambda| \leq 1 / q^{2} .
$$

On taking $y=x$ in this theorem, we get the result of Ghosh [4] and of Harman [5]:

Corollary 1. The estimate

$$
\sum_{n \leq x} \Lambda(n) e\left(n^{2} \alpha\right) \ll x^{1+\varepsilon}\left(\frac{1}{q}+\frac{1}{x^{1 / 2}}+\frac{q}{x^{2}}\right)^{1 / 4}
$$

holds for $\alpha$ satisfying $|\lambda| \leq q^{-2}$.

The estimate for $\alpha \in E_{1}$ is given in the following theorem, where GRH is introduced because, in some applications, one has to take $P$ as large as a certain positive power of $x$.

TheOREm 3. Assume GRH. Then for $\alpha \in E_{1}$ we have

$$
S_{2}(x, y ; \alpha)=M_{2}(x, y ; \alpha)+E(x, y),
$$

where $M_{2}(x, y ; \alpha)$ is defined as in $(1.7)$, and

$$
E(x, y) \ll x^{1 / 2+\varepsilon}\left(P^{1 / 2}+\frac{y}{Q^{1 / 2}}+\frac{y x^{1 / 2}}{Q}\right) .
$$

Throughout this paper, we will use all the notations introduced in this section. In addition, the letter $c$ without subscript stands for an absolute 
positive constant, while $\delta$ is an arbitrary positive constant; both of them may be different at each occurrence. For example, we may write

$$
(\log x)^{c}(\log x)^{c} \ll(\log x)^{c}, \quad x^{\delta}(\log x)^{c} \ll x^{\delta} .
$$

2. Proof of Theorem 2. Let $a_{m}, b_{n}$ be any real numbers satisfying $a_{m} \ll \tau^{c}(m), b_{n} \ll \tau^{c}(n)$, where $\tau(n)$ is the divisor function. In addition to the condition of Theorem 2, we assume without loss of generality that $y \geq x^{1 / 2}$ throughout this section; the theorem is trivial otherwise.

The proof of Theorem 2 depends on Vaughan's identity and the two propositions below. In the proof of the propositions the following estimates (see [3] for example) will be used several times:

$$
\begin{gathered}
\tau^{c}(n) \ll n^{\delta} ; \\
\sum_{n \leq Y} \min \left(X, \frac{1}{\|n \alpha\|}\right) \ll\left(\frac{Y}{q}+1\right)(X+q \log q),
\end{gathered}
$$

where $\|u\|$ denotes the smallest distance between $u$ and all integers.

Proposition A. Let $M, N \geq 1, x \ll M N \ll x$ and

$$
J_{1}=\sum_{m \sim M} \sum_{\substack{n \sim N \\ x<m n \leq x+y}} a_{m} b_{n} e\left(m^{2} n^{2} \alpha\right),
$$

where $m \sim M$ and $n \sim N$ denote $M<m \leq 2 M$ and $N<n \leq 2 N$ respectively. Then

$$
J_{1} \ll y^{1+\varepsilon}\left(\frac{1}{q}+\frac{M}{y}+\frac{x^{2}}{M^{2} y^{2}}+\frac{q x}{y^{3}}\right)^{1 / 4} .
$$

P r o of. Without loss of generality we assume that $M \leq x^{1 / 2}$. By Cauchy's inequality and (2.1) we obtain

$$
\begin{aligned}
\left|J_{1}\right|^{2} & \ll N^{1+\delta} \sum_{n \sim N}\left|\sum_{\substack{m \sim M \\
x<n m \leq x+y}} a_{m} e\left(m^{2} n^{2} \alpha\right)\right|^{2} \\
= & N^{1+\delta} \sum_{m_{1} \sim M} \sum_{\substack{m_{2} \sim M \\
m_{1} \neq m_{2}}} a_{m_{1}} a_{m_{2}} \sum_{\substack{n \sim N \\
x<n m_{i} \leq x+y}} e\left(n^{2}\left(m_{1}^{2}-m_{2}^{2}\right) \alpha\right)+O\left(N y^{1+\delta}\right) \\
\ll & N^{1+\delta} \sum_{m_{1} \sim M} \sum_{\substack{m_{2} \sim M \\
0<m_{1}-m_{2} \ll y N^{-1}}}\left|\sum_{\substack{n \sim N \\
x<n m_{i} \leq x+y}} e\left(n^{2}\left(m_{1}-m_{2}\right)\left(m_{1}+m_{2}\right) \alpha\right)\right| \\
& +N y^{1+\delta} .
\end{aligned}
$$


Let $r=m_{1}-m_{2}, m_{2}=m$. Then $0<r \ll y N^{-1}$, and it follows that

$$
\left|J_{1}\right|^{2} \ll N^{1+\delta} \sum_{0<r \ll y N^{-1}} \sum_{m \sim M}\left|\sum_{\substack{n \sim N \\ \frac{x}{m}<n \leq \frac{x+y}{m+r}}} e\left(n^{2} r(2 m+r) \alpha\right)\right|+N y^{1+\delta} .
$$

Applying Cauchy's inequality once again, by (2.1) we get

$$
\begin{aligned}
\left|J_{1}\right|^{4} \ll x^{1+\delta} y & \sum_{0<r \ll y N^{-1}} \sum_{m \sim M}\left|\sum_{\substack{n \sim N \\
m \sim n \\
\frac{x}{m}<n \leq \frac{x+y}{m+r}}} e\left(n^{2} r(2 m+r) \alpha\right)\right|^{2}+N^{2} y^{2+\delta} \\
= & x^{1+\delta} y \sum_{0<r \ll y N^{-1}} \sum_{\substack{m \sim M \\
m}} \sum_{\substack{n_{1} \sim N \\
\frac{x}{m}<n_{1} \leq \frac{x+y}{m+r}}} \sum_{\substack{n_{2} \sim N, n_{2} \neq n_{1} \\
\frac{x}{m}<n_{2} \leq \frac{x+y}{m+r}}} e\left(r(2 m+r)\left(n_{1}^{2}-n_{2}^{2}\right) \alpha\right) \\
& +O\left(x y^{3+\delta} N^{-1}\right)+O\left(N^{2} y^{2+\delta}\right) .
\end{aligned}
$$

Let $k=n_{1}-n_{2} \ll y M^{-1}, n_{1}=n$. It follows that

$$
\begin{aligned}
& \left|J_{1}\right|^{4} \ll x^{1+\delta} y \sum_{0<r \ll y N^{-1}} \sum_{0<k \ll y M^{-1}} \sum_{m \sim M}\left|\sum_{\substack{n \sim N \\
N_{1}<n \leq N_{2}}} e(r(2 m+r) k(2 n+k) \alpha)\right| \\
& \quad+x y^{3+\delta} N^{-1}+N^{2} y^{2+\delta} \\
& \ll x^{1+\delta} y \sum_{0<r \ll y N^{-1}} \sum_{0<k \ll y M^{-1}} \sum_{m \sim M}\left|\sum_{\substack{n \sim N \\
N_{1}<n \leq N_{2}}} e(2 r k(2 m+r) \alpha)\right| \\
& \quad+x y^{3+\delta} N^{-1}+N^{2} y^{2+\delta},
\end{aligned}
$$

where

$$
N_{1}=\frac{x}{m}, \quad N_{2}=\frac{x+y}{m+r}-k, \quad N_{2}-N_{1} \ll \frac{y}{M} .
$$

Then by (2.2) we obtain

$$
\begin{aligned}
\left|J_{1}\right|^{4} & \ll x^{1+\delta} y \sum_{r} \sum_{k} \sum_{m} \min \left(\frac{y}{M}, \frac{1}{\|2 r k(2 m+r) \alpha\|}\right)+x y^{3+\delta} N^{-1}+N^{2} y^{2+\delta} \\
& \ll x^{1+\delta} y \sum_{0<n \ll y^{2} N^{-1}} \tau^{c}(n) \min \left(\frac{y}{M}, \frac{1}{\|n \alpha\|}\right)+x y^{3+\delta} N^{-1}+N^{2} y^{2+\delta} \\
& \ll y^{4+\delta}\left(\frac{1}{q}+\frac{M}{y}+\frac{x^{2}}{M^{2} y^{2}}+\frac{q x}{y^{3}}\right) .
\end{aligned}
$$

This proves the desired result.

Proposition B. Let $x \ll M N \ll x$, and

$$
J_{2}=\sum_{m \sim M} a_{m} \sum_{\substack{n \sim N \\ x<m n \leq x+y}} e\left(m^{2} n^{2} \alpha\right) .
$$


If $\alpha$ satisfies $|\lambda| \ll q^{-2}$, then

$$
J_{2} \ll y^{1+\varepsilon}\left(\frac{1}{q}+\frac{M^{2}}{y}+\frac{q}{y^{2}}\right)^{1 / 2} .
$$

Proof. By squaring out $\left|J_{2}\right|$, we get

$$
\begin{aligned}
\left|J_{2}\right|^{2}= & \sum_{m_{1} \sim M} a_{m_{1}} \sum_{m_{2} \sim M} a_{m_{2}} \\
& \times \sum_{\substack{n_{1} \sim N \\
x<m_{1} n_{1} \leq x+y}} \sum_{\substack{n_{2} \sim N, x<m_{2} n_{2} \leq x+y \\
m_{1} n_{1} \neq m_{2} n_{2}}} e\left(\left(m_{1} n_{1}-m_{2} n_{2}\right)\left(m_{1} n_{1}+m_{2} n_{2}\right) \alpha\right) \\
& +O\left(y^{1+\delta}\right) .
\end{aligned}
$$

Let $r=m_{1} n_{1}-m_{2} n_{2}, d=\left(m_{1}, m_{2}\right), m_{1}=d \mu_{1}, m_{2}=d \mu_{2}$. Then $\left(\mu_{1}, \mu_{2}\right)=$ $1, d \mid r$ and $d \ll M$. Write $r=\varrho d$. It follows that $\mu_{i} \sim M d^{-1}$ and $\varrho \leq y d^{-1}$ for $r>0$. By (2.1) we obtain

$$
\begin{aligned}
& \left|J_{2}\right|^{2} \ll x^{\delta} \sum_{d \ll M} \sum_{0<\varrho \ll y d^{-1}} \sum_{\mu_{1} \sim M d^{-1}} \sum_{\mu_{2} \sim M d^{-1}}\left|\sum_{n_{2}} e\left(2 d^{2} \varrho \mu_{2} n_{2} \alpha\right)\right| \\
& +y^{1+\delta},
\end{aligned}
$$

where the summation $\sum_{n_{2}}$ is taken over

$$
N_{1}<n_{2} \leq N_{2}, \quad n_{2} \equiv-\varrho \bar{\mu}_{2}\left(\bmod \mu_{1}\right),
$$

with $\bar{\mu}_{2}$ satisfying $\bar{\mu}_{2} \mu_{2} \equiv 1\left(\bmod \mu_{1}\right)$ and

$$
\begin{aligned}
& N_{1}=\max \left(\frac{x}{d \mu_{1}}, \frac{x}{d \mu_{2}}, \frac{\mu_{1} N-\varrho}{\mu_{2}}\right), \\
& N_{2}=\min \left(\frac{x+y}{d \mu_{1}}, \frac{x+y}{d \mu_{2}}, \frac{2 \mu_{1} N-\varrho}{\mu_{2}}\right), \\
& N_{2}-N_{1} \ll y M^{-1} .
\end{aligned}
$$

It therefore follows from (2.3) that

$$
\ll x^{\delta} \sum_{d \leq M}^{\left|J_{2}\right|^{2}} \sum_{0<\varrho \leq y d^{-1}} \sum_{\mu_{1} \sim M d^{-1}} \sum_{\mu_{2} \sim M d^{-1}}\left|\sum_{N_{1}^{\prime}<n \leq N_{2}^{\prime}} e\left(2 d^{2} \mu_{1} \mu_{2} \varrho n \alpha\right)\right|+y^{1+\delta},
$$

where

$$
N_{1}^{\prime}=\frac{N_{1}+\varrho \bar{\mu}_{2}}{\mu_{1}}, \quad N_{2}^{\prime}=\frac{N_{2}+\varrho \bar{\mu}_{2}}{\mu_{1}}, \quad N_{2}^{\prime}-N_{1}^{\prime} \ll d y M^{-2} .
$$

Consequently, 


$$
\begin{aligned}
\left|J_{2}\right|^{2} \ll & x^{\delta} \sum_{d \ll M} \sum_{0<\varrho \leq y d^{-1}} \sum_{\mu_{1} \sim M d^{-1}} \sum_{\mu_{2} \sim M d^{-1}} \min \left(\frac{d y}{M^{2}}, \frac{1}{\left\|2 d^{2} \mu_{1} \mu_{2} \varrho \alpha\right\|}\right) \\
& +y^{1+\delta} \\
\ll & x^{\delta} \sum_{D} \sum_{d \sim D} \sum_{0<\varrho \leq y d^{-1}} \sum_{\mu_{1} \sim M d^{-1}} \sum_{\mu_{2} \sim M d^{-1}} \min \left(\frac{D y}{M^{2}}, \frac{1}{\left\|2 d^{2} \mu_{1} \mu_{2} \varrho \alpha\right\|}\right) \\
& +y^{1+\delta},
\end{aligned}
$$

where $\sum_{D}$ is taken over $D=2^{-j} M, j \ll \log x$. From (2.1) and (2.2) it follows that

$$
\begin{aligned}
\left|J_{2}\right|^{2} & \ll x^{\delta} \sum_{D} \sum_{n \ll y M^{2} D^{-1}} \tau^{c}(n) \min \left(\frac{D y}{M^{2}}, \frac{1}{\|n \alpha\|}\right)+y^{1+\delta} \\
& \ll x^{\delta} \sum_{D}\left(\frac{y^{2}}{q}+\frac{y M^{2}}{D}+\frac{D y}{M^{2}}+q\right)+y^{1+\delta} \\
& \ll x^{\delta}\left(\frac{y^{2}}{q}+y M^{2}+q\right) .
\end{aligned}
$$

This proves the proposition.

Proof of Theorem 2. For $x^{1 / 3} \leq M \leq x^{1 / 2}$, we have

$$
\frac{M}{y}+\frac{x^{2}}{M^{2} y^{2}} \ll \frac{x^{1 / 2}}{y}+\frac{x^{4 / 3}}{y^{2}} .
$$

Thus Proposition A gives

$$
J_{1} \ll y^{1+\varepsilon}\left(\frac{1}{q}+\frac{x^{1 / 2}}{y}+\frac{x^{4 / 3}}{y^{2}}+\frac{q x}{y^{3}}\right)^{1 / 4} .
$$

While for $M \leq x^{1 / 3}$, one deduces from Proposition B that

$$
\begin{aligned}
J_{2} & \ll y^{1+\varepsilon}\left(\frac{1}{q}+\frac{x^{2 / 3}}{y}+\frac{q}{y^{2}}\right)^{1 / 2} \ll y^{1+\varepsilon}\left(\frac{1}{q^{2}}+\frac{x^{4 / 3}}{y^{2}}+\frac{q^{2}}{y^{4}}\right)^{1 / 4} \\
& \ll y^{1+\varepsilon}\left(\frac{1}{q}+\frac{x^{1 / 2}}{y}+\frac{x^{4 / 3}}{y^{2}}+\frac{q x}{y^{3}}\right)^{1 / 4},
\end{aligned}
$$

since $1 \leq q \leq x y$. The theorem now follows from Vaughan's identity [12].

3. Proof of Theorem 3. To prove the theorem, we need the following lemma, whose proof may be found in Titchmarsh [11], Lemmas 4.3 and 4.4.

Lemma 3.1. Suppose that $H(x)$ and $F(x)$ are real functions defined in $[a, b], H(x)$ and $1 / F^{\prime}(x)$ are monotonic, $|H(x)| \leq M$. 
(1) If $F^{\prime}(x) \geq m>0$, or $F^{\prime}(x) \leq-m<0$, then

$$
\int_{a}^{b} H(x) e(F(x)) d x \ll M / m .
$$

(2) If $F^{\prime \prime}(x) \geq r>0$, or $F^{\prime \prime}(x) \leq-r<0$, then

$$
\int_{a}^{b} H(x) e(F(x)) d x \ll M / \sqrt{r} \text {. }
$$

We now give

Proof of Theorem 3. We start by transforming the exponential sum $S_{2}(x, y ; \alpha)$ as follows:

$$
\begin{aligned}
& S_{2}(x, y ; \alpha) \\
& \quad=\frac{1}{\varphi(q)} \sum_{\chi \bmod q} \sum_{\substack{h=1 \\
(h, q)=1}}^{q} \bar{\chi}(h) e\left(\frac{a h^{2}}{q}\right) \sum_{x<n \leq x+y} \chi(n) \Lambda(n) e\left(n^{2} \alpha\right),
\end{aligned}
$$

where $\chi$ is a Dirichlet character $\bmod q$. Under GRH, the Siegel-Walfisz theorem takes the form (see [3])

$$
\psi(x ; \chi)=\sum_{n \leq x} \chi(n) \Lambda(n)=E(\chi) x-\sum_{|\gamma| \leq T} \frac{x^{\varrho}}{\varrho}+O\left(\frac{x L^{2}}{T}+L\right),
$$

where $E\left(\chi_{0}\right)=1, E(\chi)=0$ for other $\chi$, and $\varrho=1 / 2+i \gamma$ denotes a non-trivial zero of $\zeta(s)$. Take $T=x$, it follows from partial summation that the innermost sum in (3.1) is

$$
\int_{x}^{x+y} e\left(\lambda u^{2}\right) d u-\sum_{|\gamma| \leq x} \int_{x}^{x+y} u^{\varrho-1} e\left(\lambda u^{2}\right) d u+O\left(|\lambda| y x L^{2}+L^{2}\right) .
$$

The contribution of the first term in $(3.2)$ to $S_{2}(x, y ; \alpha)$ is $M_{2}(x, y ; \alpha)$. Appealing to Vinogradov's bound (see [13]):

$$
\left|\sum_{\substack{h=1 \\(h, q)=1}}^{q} \chi(h) e\left(\frac{a h^{2}}{q}\right)\right| \ll q^{1 / 2+\delta},
$$

the $O$-term of (3.2) contributes to $S_{2}(x, y ; \alpha)$ in an amount

$$
\ll q^{1 / 2+\delta}\left(|\lambda| y x L^{2}+L^{2}\right) \ll \frac{y x L^{2}}{Q}+P^{1 / 2+\delta},
$$

which is acceptable in (1.16). The theorem thus reduces to

$$
q^{1 / 2+\delta} \sum_{|\gamma| \leq x} \int_{x}^{x+y} u^{\varrho-1} e\left(\lambda u^{2}\right) d u \ll x^{1 / 2+\varepsilon}\left(P^{1 / 2}+\frac{y}{Q^{1 / 2}}\right) .
$$


Denote by $J$ the sum above. Then

$$
\begin{aligned}
J & \ll \sum_{|\gamma| \leq x}\left|\int_{x}^{x+y} u^{-1 / 2} e\left(\lambda u^{2}+\frac{\gamma}{2 \pi} \log u\right) d u\right| \\
& =\frac{1}{2} \sum_{|\gamma| \leq x}\left|\int_{x^{2}}^{(x+y)^{2}} v^{-3 / 4} e\left(\lambda v+\frac{\gamma}{4 \pi} \log v\right) d v\right| .
\end{aligned}
$$

Let $V$ denote the interval $\left[x^{2},(x+y)^{2}\right]$, and

$$
f(u)=\lambda v+\frac{\gamma}{4 \pi} \log v, \quad v \in V
$$

Then we have

$$
f^{\prime}(u)=\lambda+\frac{\gamma}{4 \pi v} \gg \frac{\min _{v \in V}|\gamma+4 \pi \lambda v|}{x^{2}}, \quad-f^{\prime \prime}(v)=\frac{\gamma}{4 \pi v^{2}} \gg \frac{|\gamma|}{x^{4}} .
$$

Hence by Lemma 3.1, the last integral above is

$$
\ll x^{-3 / 2} \min \left(x y, \frac{x^{2}}{\min _{v \in V}|\gamma+4 \pi \lambda v|}, \frac{x^{2}}{\sqrt{|\gamma|}}\right) .
$$

Consequently,

$$
J \ll x^{-1 / 2} \sum_{|\gamma| \leq x} \min \left(y, \frac{x}{\min _{v \in V}|\gamma+4 \pi \lambda v|}, \frac{x}{\sqrt{|\gamma|}}\right) .
$$

Take $H=32 \pi|\lambda| y x+1$, so that the inequality

$$
\left|\gamma+4 \pi \lambda x^{2}\right| \geq j H, \quad j \geq 1,
$$

implies that, for $v \in V$,

$$
|\gamma+4 \pi \lambda v| \geq j H-4 \pi|\lambda|\left((x+y)^{2}-x^{2}\right) \geq \frac{1}{2} j H .
$$

Hence (3.4) becomes

$$
\begin{aligned}
J & \ll x^{-1 / 2} \sum_{\left|\gamma+4 \pi \lambda x^{2}\right| \leq H} \min \left(y, \frac{x}{\sqrt{|\gamma|}}\right)+x^{-1 / 2} \sum_{1 \leq j \leq J_{0}} \sum_{\substack{|\gamma| \leq x \\
j H<\left|\gamma+4 \pi \lambda x^{2}\right| \leq(j+1) H}} \frac{x}{j H} \\
& \ll x^{-1 / 2} \sum_{\left|\gamma+4 \pi \lambda x^{2}\right| \leq H} \min \left(y, \frac{x}{\sqrt{|\lambda| x^{2}}}\right)+x^{1 / 2} \sum_{1 \leq j \leq J_{0}} \frac{1}{j} \sum_{\substack{|\gamma| \leq x \\
j H<\left|\gamma+4 \pi \lambda x^{2}\right| \leq(j+1) H}} 1 \\
& \ll x^{-1 / 2} \sum_{\left|\gamma+4 \pi \lambda x^{2}\right| \leq H} \min \left(y, \frac{1}{\sqrt{|\lambda|}}\right)+x^{1 / 2+\delta},
\end{aligned}
$$

where we have used the fact that $H \gg 1$ and $J_{0} \ll x^{2}$. 
We now consider two cases $|\lambda| \geq y^{-2}$ and $|\lambda|<y^{-2}$ separately. If $|\lambda| \geq$ $y^{-2}$, then

$$
J \ll x^{-1 / 2}|\lambda|^{-1 / 2} H+x^{1 / 2+\delta} \ll|\lambda|^{1 / 2} y x^{1 / 2}+x^{1 / 2+\delta} .
$$

While for $|\lambda|<y^{-2}$, one can easily deduce that

$$
J \ll x^{-1 / 2} y H+x^{1 / 2+\delta} \ll x^{-1 / 2} y(|\lambda| y x+1)+x^{1 / 2+\delta} \ll x^{1 / 2+\delta} .
$$

We thus conclude that $J \ll|\lambda|^{1 / 2} y x^{1 / 2}+x^{1 / 2+\delta}$, which, when inserted into (3.3), gives the desired result. This completes the proof of the theorem.

4. Proof of Theorem 1. We need the following lemmas.

LEMma 4.1. Let $|\alpha| \leq 1 / 2,1 \leq y \leq x$ and

$$
T_{2}(x, y ; \alpha)=\sum_{x<n \leq x+y} n^{-1 / 2} e(n \alpha) .
$$

Then

$$
T_{2}(x, y ; \alpha) \ll x^{-1 / 2} \min \left(y,|\alpha|^{-1}\right) .
$$

Lemma 4.2. Let $C(a, q)$ be defined as in Theorem 1. Then

$$
\sum_{q \leq P} \frac{1}{\varphi^{5}(q)} \sum_{\substack{a=1 \\(a, q)=1}}^{q} C^{5}(a, q) e\left(-\frac{a N}{q}\right)=\sigma(N)+O\left(P^{-1 / 2+\varepsilon}\right),
$$

where the singular series $\sigma(N)$, defined as in Theorem 1 , is convergent, and $\sigma(N)>c>0$ for $N \equiv 5(\bmod 24)$.

The first lemma can be easily verified by routine methods. For the proof of the second lemma, see [6].

LEMMA 4.3. We have

$$
\sum_{\substack{n_{1}+\ldots+n_{5}=0 \\\left|n_{j}\right| \leq U}} 1=\frac{115}{12} U^{4}+O\left(U^{3}\right) .
$$

The lemma follows readily from elementary calculations.

We now give

Proof of Theorem 1. Let

$$
U=N^{9 / 20+\varepsilon}, \quad N_{1}=\sqrt{\frac{N}{5}}-U, \quad N_{2}=\sqrt{\frac{N}{5}}+U
$$

and

$$
S_{2}(\alpha)=\sum_{N_{1}<n \leq N_{2}} \Lambda(n) e\left(n^{2} \alpha\right)
$$


Define

$$
R^{*}(N, U)=\int_{0}^{1} S_{2}^{5}(\alpha) e(-N \alpha) d \alpha
$$

It is easily seen that

$$
R^{*}(N, U)=\sum_{\substack{N=n_{1}^{2}+\ldots+n_{5}^{2} \\\left|n_{j}-\sqrt{N / 5}\right| \leq U}} \Lambda\left(n_{1}\right) \ldots \Lambda\left(n_{5}\right)=\frac{\log ^{5} N}{2^{5}} R(N, U)(1+o(1)) .
$$

The theorem therefore reduces to

$$
R^{*}(N, U)=\frac{115 \sqrt{5}}{24} \sigma(N) \frac{U^{4}}{N^{1 / 2}}(1+o(1)) .
$$

To apply the circle method, we divide the interval $[0,1]$ into major arcs $E_{1}$ and minor arcs $E_{2}$. Take

$$
P=\frac{U}{N^{1 / 4}}, \quad Q=\frac{U^{2}}{N^{1 / 4}}
$$

in (1.11), so that $E_{1}$ and $E_{2}$ are determined by (1.12). Therefore,

$$
R^{*}(N, U)=\left\{\int_{E_{1}}+\int_{E_{2}}\right\} S_{2}^{5}(\alpha) e(-N \alpha) d \alpha .
$$

For $\alpha \in E_{2}$, Theorem 2 and (1.13) now gives

$$
S_{2}(\alpha) \ll U^{1+\delta}\left(\frac{N^{1 / 4}}{U}+\frac{N^{2 / 3}}{U^{2}}\right)^{1 / 4} \ll U^{3 / 4} N^{1 / 16+\delta} .
$$

Hence the integral on $E_{2}$ is

$$
\ll\left\{\sup _{\alpha \in E_{2}}\left|S_{2}(\alpha)\right|\right\} \int_{0}^{1}\left|S_{2}(\alpha)\right|^{4} d \alpha \ll U^{3 / 4} N^{1 / 16+\delta} U^{2+\delta} \ll U^{11 / 4} N^{1 / 16+\delta},
$$

which is estimated as $o\left(U^{4} N^{-1 / 2}\right)$ since $U=N^{9 / 20+\varepsilon}$.

To evaluate the integral on $E_{1}$, we appeal to Theorem 3 , which yields

$$
\begin{aligned}
S_{2}(\alpha) & =\frac{C(a, q)}{\varphi(q)} \int_{N_{1}}^{N_{2}} e\left(\lambda u^{2}\right) d u+O\left(N^{3 / 8+\delta}\right) \\
& =\frac{C(a, q)}{2 \varphi(q)} \sum_{N_{1}^{2} \leq n \leq N_{2}^{2}} e(\lambda n) n^{-1 / 2}+O\left(N^{3 / 8+\delta}\right),
\end{aligned}
$$

where $C(a, q)$ is defined as in (4.2). Denote by $T_{2}(\lambda)$ the sum on the righthand side of (4.7). Appealing to the elementary inequality

$$
\left|a^{5}-b^{5}\right| \leq 5|a-b| \max \left(|a|^{4},|b|^{4}\right),
$$


we deduce from (4.7) that

$$
S_{2}^{5}(\alpha)-\frac{C^{5}(a, q)}{2^{5} \varphi^{5}(q)} T_{2}^{5}(\lambda) \ll N^{3 / 8+\delta}\left(\left|S_{2}(\alpha)\right|^{4}+\frac{C^{4}(a, q)}{\varphi^{4}(q)}\left|T_{2}(\lambda)\right|^{4}\right),
$$

and consequently,

$$
\begin{aligned}
& \int_{E_{1}} S_{2}^{5}(\alpha) e(-N \alpha) d \alpha \\
&-\left(\sum_{q \leq P} \sum_{\substack{a=1 \\
(a, q)=1}}^{q} \frac{C^{5}(a, q)}{2^{5} \varphi^{5}(q)} e\left(-\frac{a N}{q}\right)\right) \int_{-1 /(q Q)}^{1 /(q Q)} T_{2}^{5}(\lambda) e(-N \lambda) d \lambda \\
& \ll N^{3 / 8+\delta}\left(\int_{0}^{1}\left|S_{2}(\alpha)\right|^{4} d \alpha+\sum_{q \leq P} \sum_{\substack{a=1 \\
(a, q)=1}}^{q} \frac{C^{4}(a, q)}{\varphi^{4}(q)} \int_{0}^{1}\left|T_{2}(\lambda)\right|^{4} d \lambda\right) \\
& \ll N^{3 / 8+\delta}\left(\int_{0}^{1}\left|S_{2}(\alpha)\right|^{4} d \alpha+\int_{0}^{1}\left|T_{2}(\lambda)\right|^{4} d \lambda\right) .
\end{aligned}
$$

By Lemma 4.1,

$$
\int_{0}^{1}\left|T_{2}(\lambda)\right|^{4} d \lambda \ll \int_{0}^{1 /\left(N^{1 / 2} U\right)} U^{4} d \lambda+\int_{1 /\left(N^{1 / 2} U\right)}^{\infty}\left(N^{1 / 2} \lambda\right)^{-4} d \lambda \ll N^{-1 / 2} U^{3} .
$$

Also,

$$
\int_{0}^{1}\left|S_{2}(\alpha)\right|^{4} d \alpha \ll U^{2+\delta} .
$$

We therefore conclude that

$$
\int_{E_{1}} S_{2}^{5}(\alpha) e(-N \alpha) d \alpha
$$

$=\left(\sum_{q \leq P} \sum_{\substack{a=1 \\(a, q)=1}}^{q} \frac{C^{5}(a, q)}{2^{5} \varphi^{5}(q)} e\left(-\frac{a N}{q}\right)\right) \int_{-1 /(q Q)}^{1 /(q Q)} T_{2}^{5}(\lambda) e(-N \lambda) d \lambda+O\left(N^{3 / 8+\delta} U^{2}\right)$.

The double sum in (4.8) is, by Lemma 4.2,

$$
\frac{1}{2^{5}} \sigma(N)+O\left(P^{-1 / 2+\delta}\right)=\frac{1}{2^{5}} \sigma(N)+O\left(N^{-1 / 8+\delta} U^{-1 / 2}\right) .
$$

Appealing to Lemma 4.1 again, we obtain 


$$
\begin{aligned}
\int_{1 /(q Q)}^{1-1 /(q Q)}\left|T_{2}^{5}(\lambda)\right| d \lambda & \ll \int_{1 /(q Q)}^{\infty}\left(N^{1 / 2} \lambda\right)^{-5} d \lambda \ll(q Q)^{4} N^{-5 / 2} \\
& \ll(P Q)^{4} N^{-5 / 2} \ll U^{12} N^{-9 / 2},
\end{aligned}
$$

so that the integral on the right-hand side of (4.8) is equal to

$$
\begin{aligned}
\int_{0}^{1} T_{2}^{5}(\lambda) e(-N \lambda) d \lambda+O & \left(U^{12} N^{-9 / 2}\right) \\
& =\sum_{\substack{n_{1}+\ldots+n_{5}=N \\
N_{1}^{2} \leq n_{j} \leq N_{2}^{2}}} n_{1}^{-1 / 2} \ldots n_{5}^{-1 / 2}+O\left(U^{12} N^{-9 / 2}\right) \\
& =\left(\frac{5}{N}\right)^{5 / 2} \sum_{\substack{n_{1}+\ldots+n_{5}=N \\
N_{1}^{2} \leq n_{j} \leq N_{2}^{2}}} 1+O\left(U^{12} N^{-9 / 2}\right) .
\end{aligned}
$$

By Lemma 4.3, the last sum above is

$$
\frac{115}{12}\left(2 \sqrt{\frac{N}{5}} U\right)^{4}+O\left(N^{3 / 2} U^{3}\right)=\frac{92}{15} N^{2} U^{4}+O\left(N^{3 / 2} U^{3}\right) .
$$

Inserting these estimates into (4.8), we conclude that

$$
\int_{E_{1}} S_{2}^{5}(\alpha) e(-N \alpha) d \alpha=\frac{115 \sqrt{5}}{24} \sigma(N) U^{4} N^{-1 / 2}(1+o(1)),
$$

which, in combination with the estimate on $E_{2}$, gives (4.5), hence the theorem.

Acknowledgement. The authors are very indebted to Professor Pan Chengdong for his valuable advice and constant encouragement.

\section{References}

[1] F. C. Auluck and S. Chowla, The representation of a large number as a sum of "almost equal" squares, Proc. Indian Acad. Sci. Sect. A 6 (1937), 81-82.

[2] A. Balog and A. Perelli, Exponential sums over primes in short intervals, Acta Math. Hungar. 48 (1986), 223-228.

[3] H. Davenport, Multiplicative Number Theory, 2nd ed., revised by H. L. Montgomery, Springer, 1980.

[4] A. Ghosh, The distribution of $\alpha p^{2}$ modulo one, Proc. London Math. Soc. (3) 42 (1981), 252-269.

[5] G. Harman, Trigonometric sums over primes I, Mathematika 28 (1981), 249-254.

[6] L.-K. Hua, Some results in the additive prime number theory, Quart. J. Math. 9 (1938), 68-80. 
[7] C.-H. Jia, Three prime theorem in short intervals (VII), Acta Math. Sinica 10 (1994), 369-387.

[8] J. Y. Liu and T. Zhan, Estimation of exponential sums over primes in short intervals $(I)$, to appear.

[9] - - - Estimation of exponential sums over primes in short intervals (II), in: Proceedings of the Halberstam Conference on Analytic Number Theory, Birkhäuser, 1996, to appear.

[10] C. D. Pan and C. B. Pan, On estimations of trigonometric sums over primes in short intervals (III), Chinese Ann. Math. Ser. B 11 (1990), 138-147.

[11] E. C. Titchmarsh, The Theory of the Riemann Zeta-function, 2nd ed., revised by D. R. Heath-Brown, Oxford University Press, 1988.

[12] R. C. Vaughan, An elementary method in prime number theory, in: Recent Progress in Analytic Number Theory, H. Halberstam and C. Hooley (eds.), Academic Press, 1981, 341-348.

[13] I. M. Vinogradov, Estimation of certain trigonometric sums with prime variables, Izv. Akad. Nauk SSSR Ser. Mat. 3 (1939), 371-398 (in Russian).

[14] E. M. Wright, The representation of a number as a sum of three or four squares, Proc. London Math. Soc. 42 (1937), 481-500.

[15] T. Zhan, On the representation of large odd integers as sum of three almost equal primes, Acta Math. Sinica (N.S.) 7 (1991), 159-272.

Department of Mathematics

Shandong University

Jinan, Shandong 250100

P. R. China

E-mail: zhantao@shandong.ihep.ac.cn 